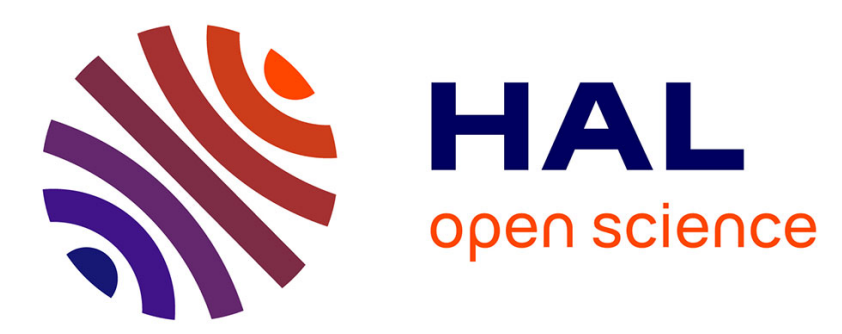

\title{
Kinetics of thermal desorption using Auger electron spectroscopy application to cesium covered (110) gallium arsenide
}

\author{
J. Derrien, F. Arnaud d'Avitaya
}

\section{- To cite this version:}

J. Derrien, F. Arnaud d'Avitaya. Kinetics of thermal desorption using Auger electron spectroscopy application to cesium covered (110) gallium arsenide. Revue de Physique Appliquée, 1976, 11 (3), pp.377-385. 10.1051/rphysap:01976001103037700 • jpa-00244071

\section{HAL Id: jpa-00244071 https://hal.science/jpa-00244071}

Submitted on 1 Jan 1976

HAL is a multi-disciplinary open access archive for the deposit and dissemination of scientific research documents, whether they are published or not. The documents may come from teaching and research institutions in France or abroad, or from public or private research centers.
L'archive ouverte pluridisciplinaire HAL, est destinée au dépôt et à la diffusion de documents scientifiques de niveau recherche, publiés ou non, émanant des établissements d'enseignement et de recherche français ou étrangers, des laboratoires publics ou privés. 


\title{
KINETICS OF THERMAL DESORPTION USING AUGER ELECTRON SPECTROSCOPY APPLICATION TO CESIUM COVERED (110) GALLIUM ARSENIDE
}

\author{
J. DERRIEN and F. ARNAUD D'AVITAYA \\ Centre de Recherche sur les Mécanismes de la Croissance Cristalline, C. N. R. S., \\ Université d'Aix-Marseille II, U. E. R. Scientifique de Luminy, Département de Physique, \\ 13288 Marseille Cedex 2, France
}

(Reçu le 5 décembre 1975, accepté le 19 janvier 1976)

\begin{abstract}
Résumé. - Nous proposons une nouvelle méthode pour suivre continûment, par Spectroscopie Auger, sous ultra-vide, les cinétiques d'ad- ou de désorption d'un corps A sur la surface d'un substrat B. L'appareillage réalisé permet de transformer un spectromètre Auger classique en suiveur de vitesse de ces réactions de surface.

Nous appliquons notre technique à une étude préliminaire de la cinétique de thermodésorption du césium déposé sur l'arseniure de gallium face (110) et mettons en évidence différents états d'adsorption.
\end{abstract}

Abstract. - We propose a new method for monitoring surface reactions kinetics using Auger Spectroscopy. It allows continuous observations of adsorption and desorption kinetics of an element $\mathrm{A}$ on the surface of a substrate $\mathrm{B}$. The apparatus designed for this purpose transforms a standard Auger electron spectrometer into a detector measuring the rate of these surface reactions.

We have used this technique to study the thermal desorption of cesium from (110) gallium arsenide. Several binding states are found.

1. Introduction. - Techniques used for detailed studies of solid surfaces under ultra high vacuum conditions (U. H. V.) are numerous and complementary. One of them, Auger Electron Spectroscopy (A. E. S.) has been developed considerably in recent years. In A. E. S., the energy distribution $N(E)$ of secondary electrons is analysed. These electrons are emitted from a solid when it is irradiated by an incident electron beam. Certain peaks, observed in the $N(E)$ spectrum, correspond to Auger transitions of the elements present at the target superficial zone. This fact is due to the small escape lengths of Auger electrons. Hence A. E.S. allows detailed studies of a solid surface.

The scope of A.E. S. applications is wide and it includes, for example, fundamental surface studies, corrosion, surface chemisorption, catalysis...

A. E. S. detects and identifies traces of most elements on the surface (less than 1/100 of a monolayer $\sim 10^{12}$ atoms $/ \mathrm{cm}^{2}$ ). With its great surface sensitivity, A. E. S. is also used to identify corrosion products, surface impurities, grain boundary impurities, embrittlement and so on. A more complete review of this technique is given in reference [1].

In this paper, we propose a new application for Auger spectroscopy : the measurement of surface reactions rates (desorption rate, condensation rate or rate of superficial compound formation). This is achieved by adding new electronic apparatus to a conventional Auger spectrometer. This simple apparatus, built in our laboratory, will be described in chapter 2 .

In chapter 3, we shall see how the thermal desorption of cesium adsorbed on the (110) gallium arsenide may be studied by means of this technique.

In a thermal desorption experiment, one usually adsorbs an initial known quantity $n$ of an element $A$ on the surface of another element B. The substrate temperature is then raised rapidly by a heating function $T(t)$ and the desorption rate $-\frac{\mathrm{d} n(\mathrm{t})}{\mathrm{d} t}$ versus time $t$ and/of temperature $T$ is recorded. The measurement of $\frac{\mathrm{dn}(\mathrm{t})}{\mathrm{d} t}$ allows one to compute $n(t)$ by integration. $n(t)$ is the quantity of A adsorbed on the surface of $\mathrm{B}$ at time $t$ during the desorption. One can thereby determine the desorption energy $E_{\mathrm{d}}$ by analysing the following equation :

$$
-\frac{\mathrm{d} n(t)}{\mathrm{d} t}=v(n) n(t) \exp -\frac{E_{\mathrm{d}}(n)}{k T(t)} .
$$

Eq. (1) is applicable in the case of a first order desorption [2]. Generally the desorption rate $-\frac{\mathrm{d} n(t)}{\mathrm{d} t}$ is detected with a mass spectrometer. 
We shall show in the next chapter that with our modified Auger spectrometer, we can measure simultaneously and continuously $\frac{\mathrm{d} n(t)}{\mathrm{d} t}$ and $n(t)$, directly on the surface.

2. Apparatus. - 2.1 Principle of A StANDARd AUGER SPECTROMETER. - We recall here briefly the function of a cylindric mirror analyser (C. M. A.) as illustrated in figure 1. Secondary electrons emitted

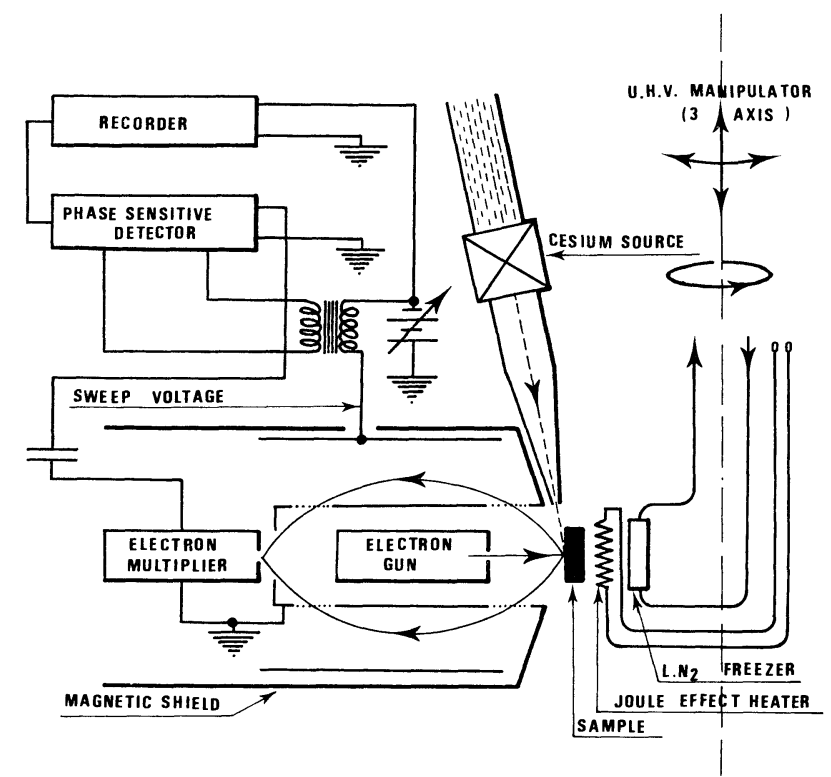

FIG. 1. - Operational principle of a commercially available C. M. A. Auger spectrometer. During our study, a metallic cesium source and a performant ultra high vacuum manipulator are also used.

from the sample are analysed in energy by a cylindric capacitor connected to a voltage $V$. Electrons with an energy $E=\mathrm{e} V$ pass through this electrostatic analyser and reach the electron multiplier. By scanning the voltage ramp $V$, one obtains the energy distribution $N(E)$. The area under the Auger peak of an element on the energy distribution curve $N(E)$ is proportional to the amount $n$ of this element. Unfortunately, $N(E)$ Auger peaks are generally buried in the large background due to all other secondary electrons. One therefore uses a phase sensitive detector (Lock in Amplifier) which gives the $\frac{\mathrm{d} N(E)}{\mathrm{d} E}$ spectrum, instead of the $N(E)$ spectrum in order to obtain a higher accuracy and an improved resolution of the peaks.

Figure 2 shows the two spectra $N(E)$ and $\frac{\mathrm{d} N(E)}{\mathrm{d} E}$, in the low energy region, obtained from a clean $\mathrm{GaAs}$ (110) face.

For a given element, the $\frac{\mathrm{d} N(E)}{\mathrm{d} E}$ peak to peak height of its Auger line is proportional to its quantity $n$

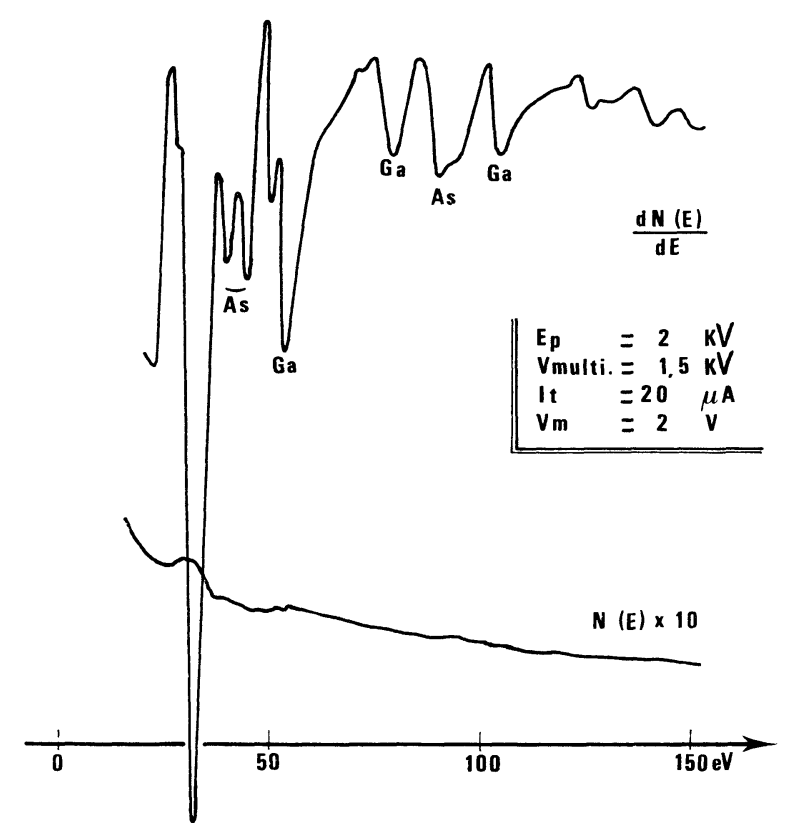

FIG. 2. - Low energy region Auger spectrum of the (110) GaAs surface after an argon sputtering and annealing cycle.

(Fig. 3a). This peak to peak value can be taken as a measure for the total Auger electron emission only in the case where the shape of Auger peak $N(E)$ is

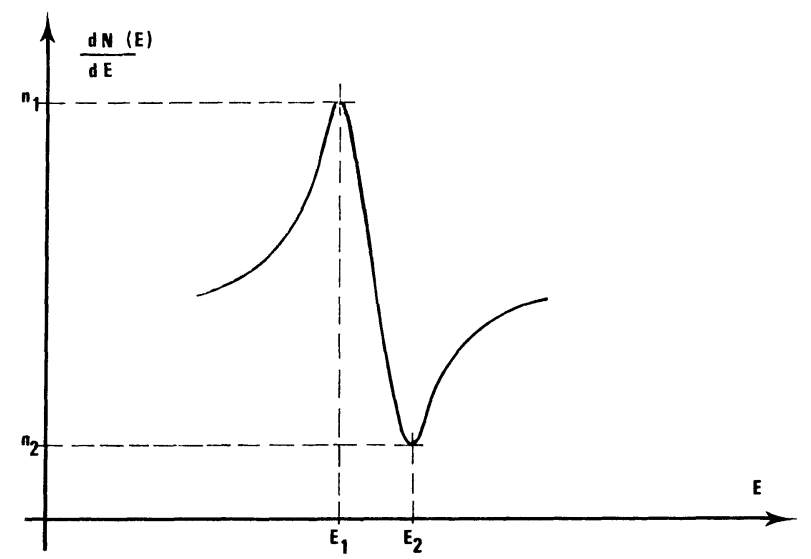

Fig. $3 a$. - The peak to peak height $\mathrm{d} N / \mathrm{d} E$ is proportional to the quantity $n$ (see ref. [3]).

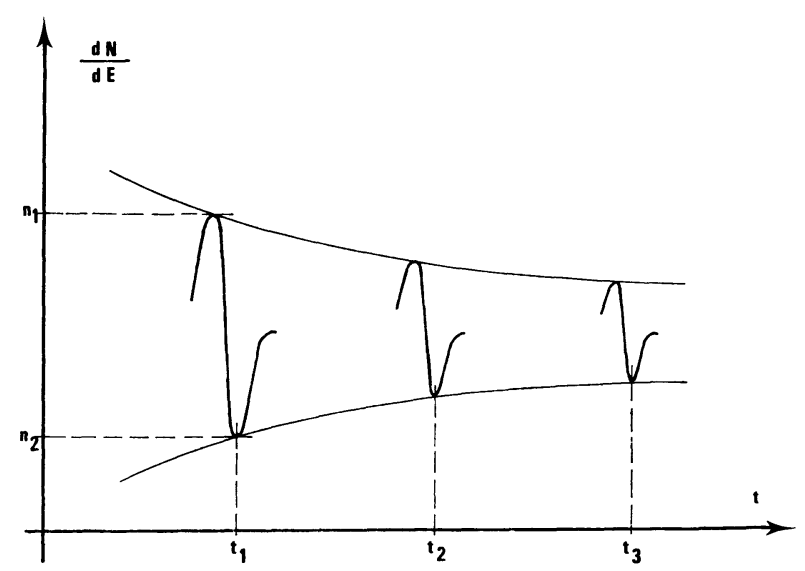

Fig. $3 b$. - The discontinuous plot of $\mathrm{d} N / \mathrm{d} E$ allows one to follow slow kinetics of the quantity $n$. 
independent of the amount of the material present [3]. For the study of dynamic processes, one monitors the quantity $n$, i. e. the $\frac{\mathrm{d} N(E)}{\mathrm{d} E}$ peak to peak height as a function of time. A typical plot of such a measurement is given in figure $3 b$. The difference between the two envelopes $n_{1}(t)$ and $n_{2}(t)$ gives

$$
n(t)=n_{1}(t)-n_{2}(t)
$$

Several studies concerning thermal desorption have used this discontinuous technique $[4,5,6,7,8]$.

The time required for two successive plots of one peak is however several seconds, even with the relatively fast C. M. A. In principle, one could work with higher scanning speeds but only at the expense of signal to noise ratio. Moreover, in the case where $n(t)$ varies rapidly with time, the number of measurable points is limited and thus the precision of $\frac{\mathrm{d} n(t)}{\mathrm{d} t}$ is quite low.

2.2 Proposed MeThod. - In a kinetic experiment, it is better to record the quantity $n(t)$ and its derivative $\frac{\mathrm{d} n(t)}{\mathrm{d} t}$ continuously. For example, in a thermal desorption experiment, one needs to measure $n(t)$ and $\frac{\mathrm{d} n(t)}{\mathrm{d} t}$ for evaluating the eq. (1). For this purpose, we propose the following method (see also Fig. 4 and 5) :

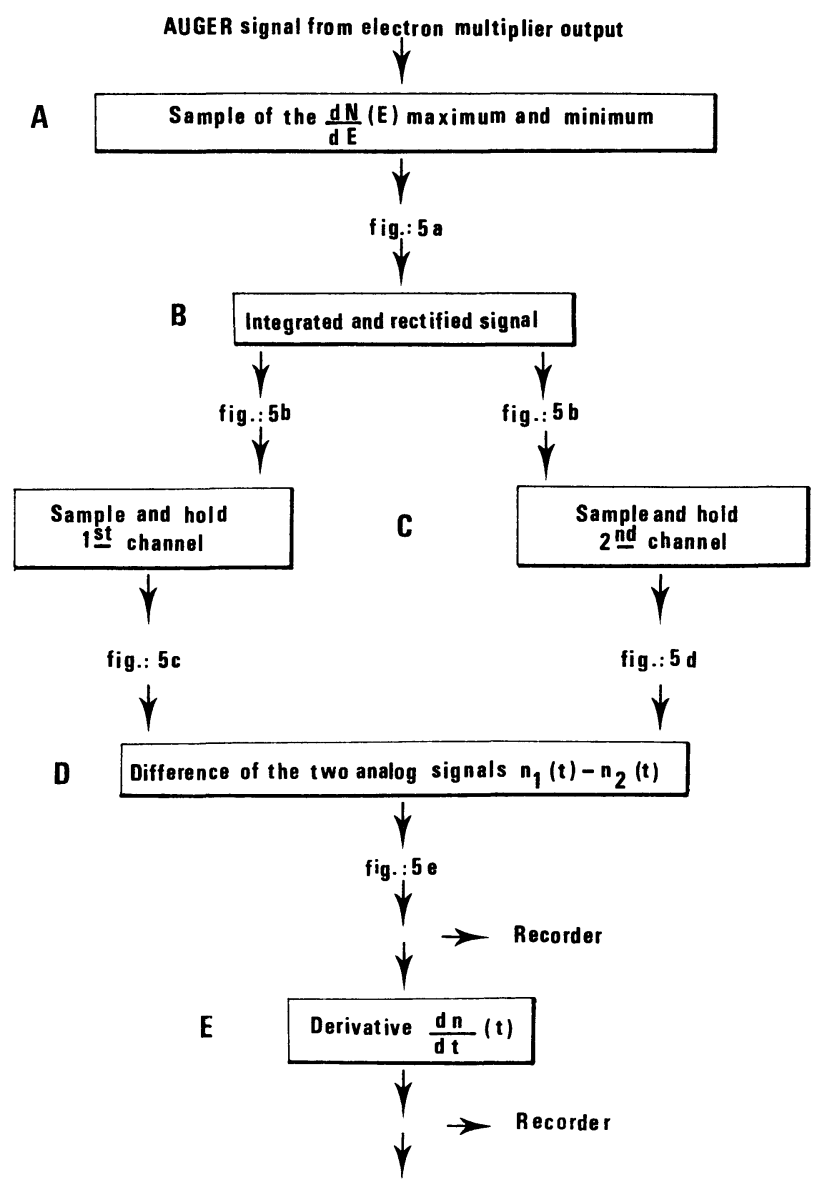

FIG. 4. - Synoptic scheme of the proposed method.
A) Instead of scanning a whole energy region around the studied Auger peak, we sample only the maximum and the minimum of the $\frac{\mathrm{d} N(E)}{\mathrm{d} E}$ Auger peak, by replacing the $V$ voltage ramp by a low frequency rectangular signal such that:

- The voltage $V_{1}$ of the rectangular signal corresponds to the maximum of the $\frac{\mathrm{d} N}{\mathrm{~d} E}$ peak i. e. $n_{1}$.

- The voltage $V_{2}$ of the rectangular signal corresponds to the minimum of the $\frac{\mathrm{d} N}{\mathrm{~d} E}$ peak i. e. $n_{2}$.

The signal, at the lock in amplifier output is then a rectangular signal whose maxima and minima give the values $n_{1}$ and $n_{2}$ of the chosen peak (Fig. 5a) and hence the quantity $n$ adsorbed on the surface.

We recall here that Sickafus and Colvin [9] have designed a more sophisticated multichannel monitor which allows one to shorten the effective scanning time of Auger Electron Spectroscopy and also to obtain a discontinuous $n(t)$ plot. The time required for two successive $n(t)$ values is, in their experiment, a few seconds. These authors have not treated these discontinuous signals $n(t)$ in order to obtain the continuous derivatives $\frac{\mathrm{d} n(t)}{\mathrm{d} t}$ (see below).

B) The signal at the lock in amplifier output is slightly integrated without loosing information with a time constant $\sim 0.03 \mathrm{~s}$, (Fig. 5b), and centered around the electrical zero. It is then rectified by an operational amplifier. The two rectified half periods of the original signal are fed into two separate channels, one containing the $n_{1}(t)$ signal, and the other the $n_{2}(t)$ signal (Fig. $5 c$ and $d$ ).

C) We sample the maximum signal value of each channel by means of a synchrone pulse. This pulse opens, with a field effect transistor, an electronic circuit which stores the sampled value and holds it until the next half period. This sample and hold circuit consists of integrated operational amplifiers and the operation is repeated on each maximum value. Hence we obtain now on the first channel a continuous signal $n_{1}(t)$ and on the second channel a continuous signal $n_{2}(t)$ (Fig. $e$ ).

D) The difference of these analog signals gives

$$
n(t)=n_{1}(t)-n_{2}(t)
$$

i. e. the quantity present on the solid surface.

E) An analog derivator then allows to follow $\frac{\mathrm{d} n(t)}{\mathrm{d} t}$ i. e. the $n(t)$ kinetics.

If the Auger signals are two low and noisy, prevision is made for the connection of calibrated linear ampli- 


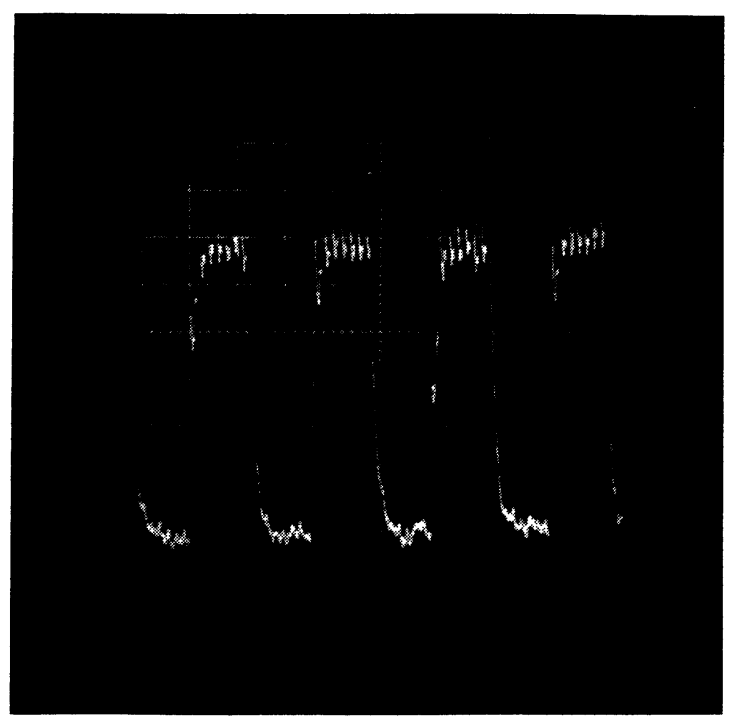

(a)

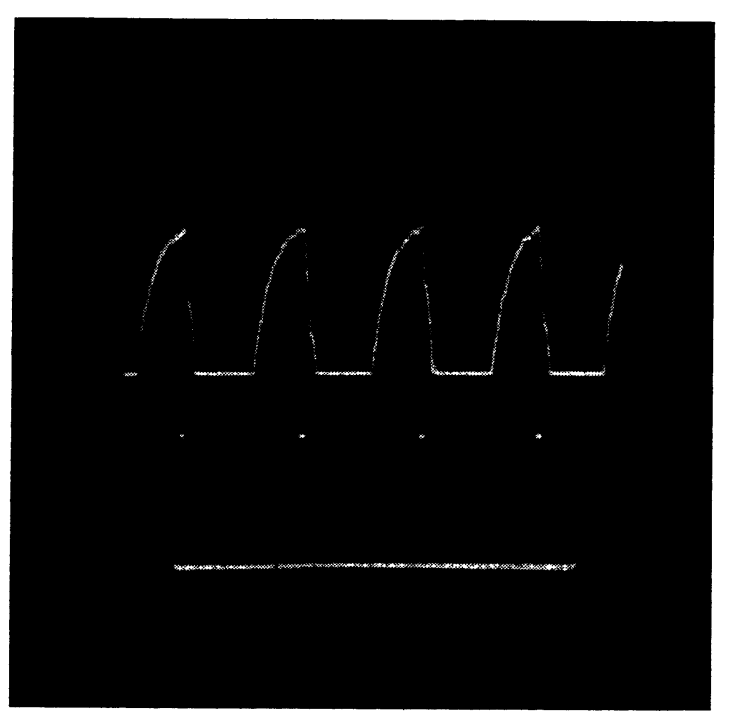

(c)

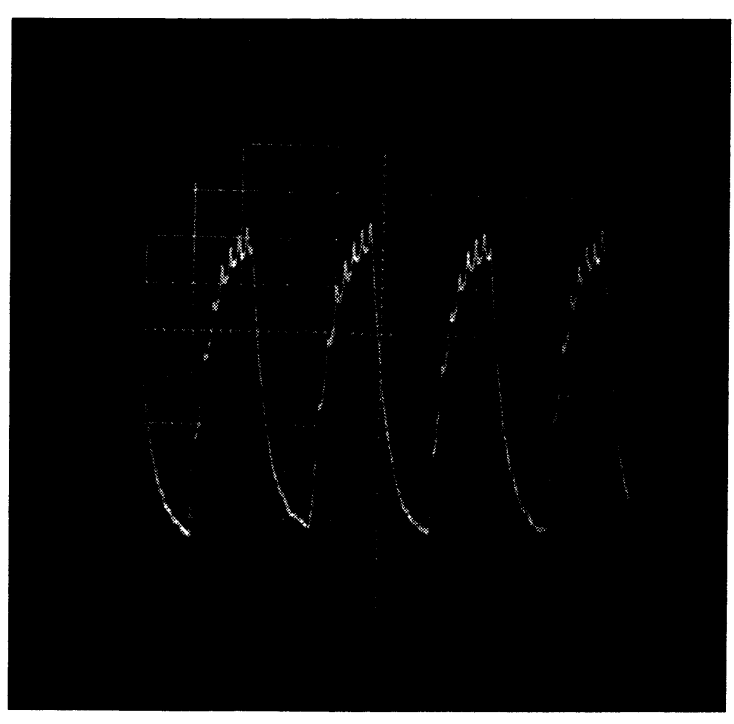

(b)

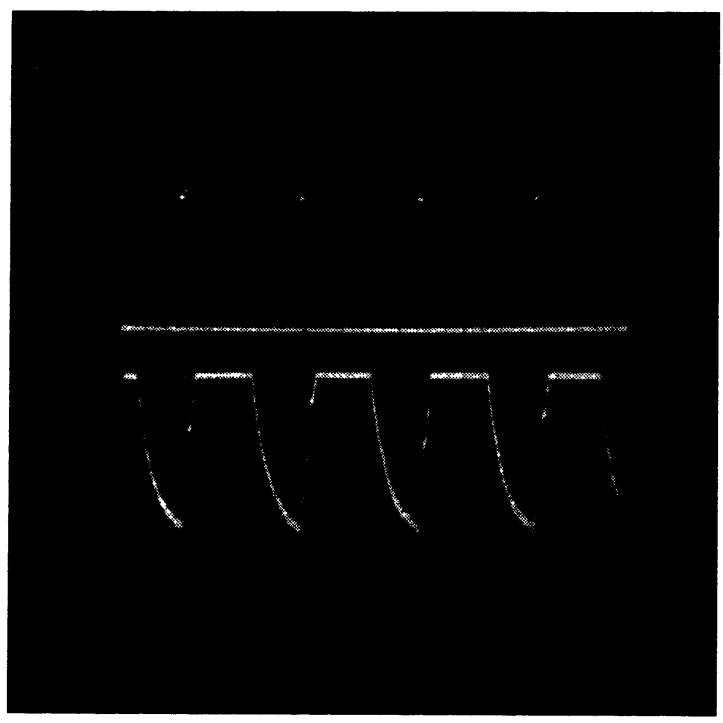

(d)

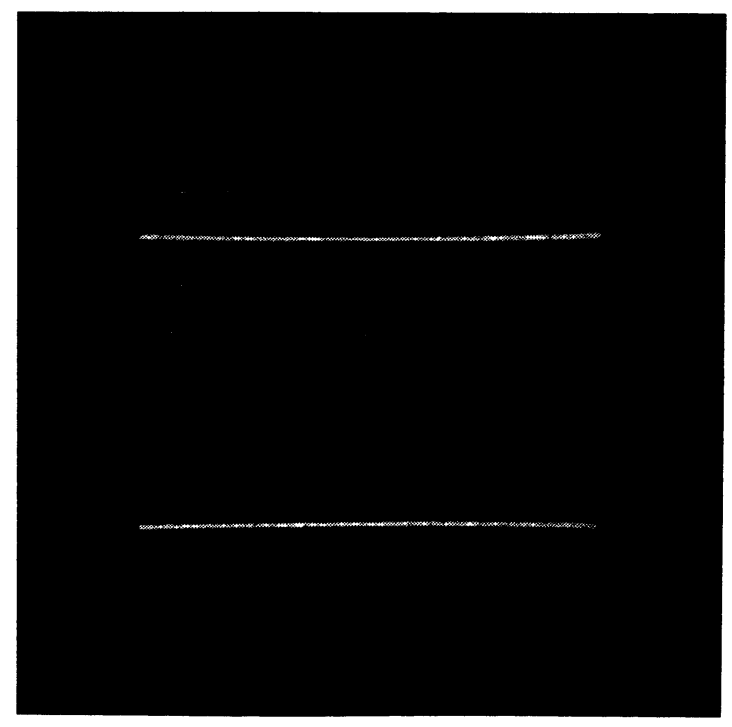

(e)

FIG. 5. - See the text. 
fiers and filters between D and E. The circuitry of the designed system is represented in figure 6.

The apparatus was examined for possible distortions by using well defined input signals (straight line, parabola, decreasing exponential, Gaussian, rectangular signal...) which were derived from an analog computer. No instrumental distorsion was observed.

As an application of our proposed method, we have studied the thermal desorption kinetics of cesiated (110) GaAs. This system has been investigated, with other surface techniques, by many laboratories and there exists some information about it.

3. Thermal desorption of cesium from the cesiated (110) gallium arsenide. - 3.1 Present CONTEXT of THE PROBLEM. - It has been known for many years that adsorption of a small quantity of $\mathrm{Cs}$ on GaAs considerably lowers the work function [10] (from $\sim 5 \mathrm{eV}$ to $\sim 1.4 \mathrm{eV}$ ). It can be further lowered by additional $\mathrm{O}_{2}$ adsorption. In this way, the $\mathrm{Cs} / \mathrm{O} / \mathrm{GaAs}$ system exhibits negative electron affinity properties [11] and is of great technological interest. New developments of surface techniques allowed several laboratories to study the Cs adsorption on III-V semiconductor compounds under U. H. V., for example by :

- Low Energy Electron Diffraction (L. E. E. D.) and Auger Electron Spectroscopy (A. E. S.) [6, 7, 12, $13,14]$.

- Work function measurements $[12,14,15,16]$.

- Photoemission $[6,7,13,16]$.

- Electron Spectroscopy for Chemical Analysis (E. S. C. A.) [17].

All these investigations permit a better detailed comprehension of adsorption but in our knowledge, only one new experiment of thermal desorption by Golstein [18] gives results directly related to the binding energies of $\mathrm{Cs}$ on (100) GaAs. This author followed the desorption kinetics with a quadripole mass spectrometer.

We present here thermal desorption results obtained with the apparatus described in chapter 2. Experiment are carried out with Cs adsorbed on (110) GaAs. We use Auger spectroscopy to measure simultaneously $\frac{\mathrm{d} n(t)}{\mathrm{d} t}$ and $n(t)$ of Cs.

3.2 Surface PReparation. - Single GaAs crystals, grown by a horizontal Bridgmann method, $\mathrm{Cr}$ doped to $7 \times 10^{17} \mathrm{~cm}^{3}$, are cleaved in air, parallel to (110) faces. The dimensions are about $5 \times 5 \times 1 \mathrm{~mm}$.

The crystal is attached to a sample holder in side a U. H. V. chamber (residual pressure $\sim 10^{-10}$ torr). The sample holder has Joule effect heating and liquid nitrogen cooling facilities as seen in figure 1. This versatile set up can be used in conjunction with several measuring probes.

The GaAs crystal is at first bombarded by Argon ions (energy $\sim 400 \mathrm{eV}$, ion current $\sim 3 \mu \mathrm{A} / \mathrm{cm}^{2}$ ) to sputter REVUe DE PHYSIQUe APPLIQUÉE. - T. 11, N॰ 3, MAI 1976. contaminants. It is then annealed at $580{ }^{\circ} \mathrm{C}$ under U. H. V. The cleanliness is controlled by standard A. E. S. No impurities were detected on the (110) face, except for a trace of carbon ( 5/100 monolayer) which increases after several thermal desorption experiments. This contaminant can only be removed by a new sputtering and annealing cycle.

L. E. E. D. of clean surfaces gives a $1 \times 1$ diagram of the stable (110) face.

3. 3 Cesium Deposition ANd CALIBRATION. - Cesium is deposited on clean surfaces. In certain experiments, it is provided by a Cs doped zeolite gun [19] and in other experiments it originates from a metallic source [20]. No difference, in thermal desorption, has been observed with the two sources.

The crystal temperature during Cs adsorption is maintained at $50^{\circ} \mathrm{C}$. Deposited quantities $n$ are controlled and calibrated by measuring, versus increasing time, the peak to peak height of the $\left(\mathrm{N}_{45} \mathrm{O}_{23} \mathrm{O}_{23}\right)$ Cs Auger transition at $47 \mathrm{eV}$.

The calibration curve shown in figure 7 has been plotted with the following Auger spectrometer conditions :

Incident energy $E_{\mathrm{p}}=2 \mathrm{kV}$.

Target current $I_{\mathrm{t}}=20 \mu \mathrm{A}$.

Electron Multiplier voltage $V_{\mathrm{ME}}=1.5 \mathrm{kV}$.

Modulation voltage $V_{\mathrm{M}}=2 \mathrm{~V}$ peak to peak.

The geometry of figure 1 allows A. E.S. to be carried out simultaneously during adsorption for a constant Cs arrival rate. The points, shown in figure 7, are obtained by standard Auger Spectroscopy at regular time intervals. The continuous line was obtained with our modified Auger system. Figure 7 demonstrates that our method can also be used to follow adsorption kinetics.

The calibration curve has two well marked linear segments, and an asymptotic behaviour at long deposition time. This saturation corresponds to the adsorption equilibrium for the given Cs incident flux and substrate temperature. The first linear segment had been also observed by Van Bommel et al. [13]. It confirms the proportionality between the Auger peak to peak height and the deposited quantity $n$, assuming that the sticking coefficient, i. e. the ratio of deposited quantity over incident quantity, remains constant during the initial stages of growth. This fact was pointed out by Smith et al. [17] and by Van Bommel et al. [13]. At the deflection point the number of Cs adsorbed atoms is about $4.6 \times 10^{14}$ atoms $/ \mathrm{cm}^{2}$. This value was obtained by Van Bommel et al. [13] by an analysis of the L. E. E. D. $(4 \times 4)$ Cs superstructure on (110) GaAs. These authors also calibrated their Cs incident flux with a Langmuir Taylor probe. We have also deposited cesium from the zeolite gun. The Cs absolute coverage is determined from time integral Cs ion current, measured directly from the sample. We assume that the sticking coefficient is equal to unity. 


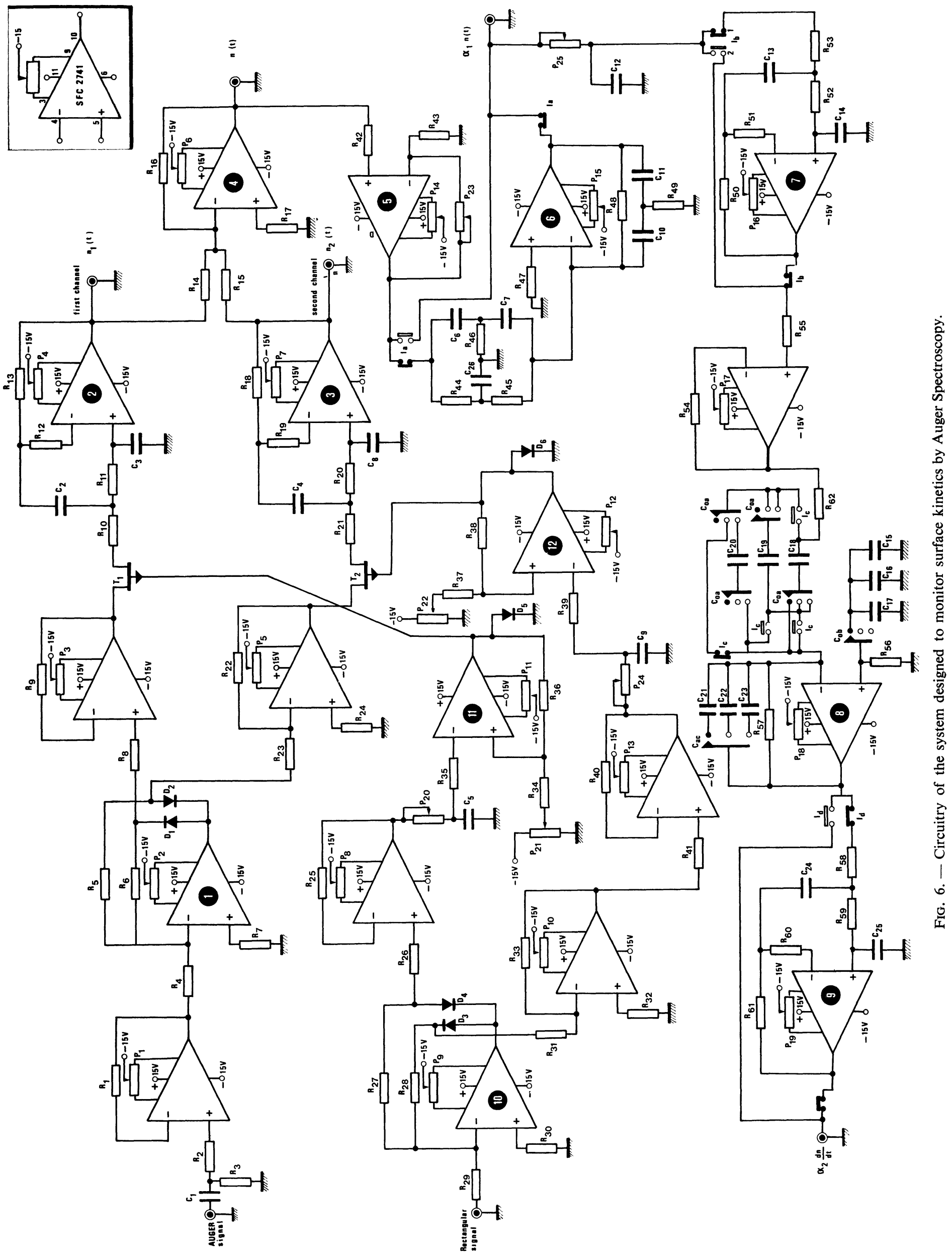


Effects of migration, reflection, are not taken in account in the calculation. We then verify that, at the deflection point, the number of Cs adsorbed atoms is really $4.6 \times 10^{14}$ atoms $/ \mathrm{cm}^{2}$ within experimental errors.

This value will be taken as an absolute reference for our Auger calibration. We shall denote this quantity as a monolayer.

We observe a second linear segment on our curve and we interpret it as a second layer of Cs which probably grows on top of the first one.

Auger electrons have small escape lengths. The contribution of the first layer to the Auger peak

FIG. 6 (suite)

\section{CIRCUITRY PARTS LIST (see Fig. 6)}

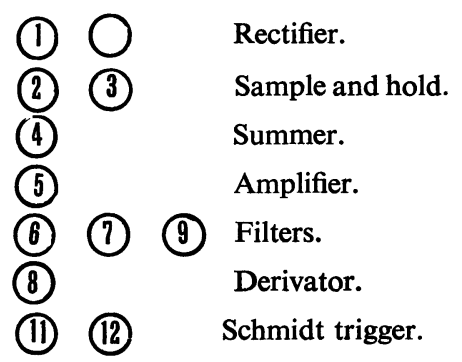

DIODES All diodes are SILEC F6 Z

Capacitor $C_{n}$.

\begin{tabular}{c|l} 
Values $(\mu \mathrm{F})$ & \multicolumn{1}{|c}{$n$} \\
0.01 & 23 \\
0.047 & $6,7,10,11,22$ \\
0.1 & 26 \\
1 & 21 \\
5 & 13,24 \\
10 & $3,8,14$ to 20,25 \\
22 & 2,4 \\
100 & $1,5,9$
\end{tabular}

Potentiometer $\boldsymbol{P}_{n}$.

\begin{tabular}{c|l} 
Values $(k \Omega)$ & \multicolumn{1}{|c}{$n$} \\
5 & 20,24 \\
10 & 1 to 19 \\
30 & 21 \\
50 & 22,25 \\
100 & 23
\end{tabular}

Resistor $R_{n}$.

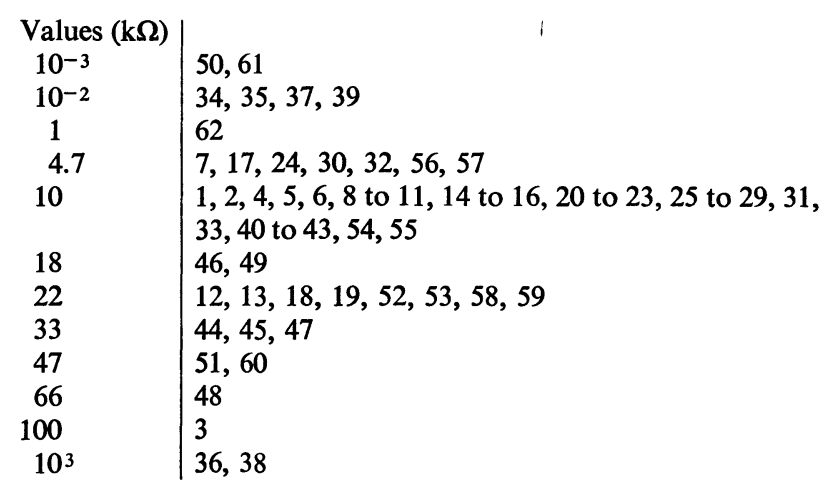

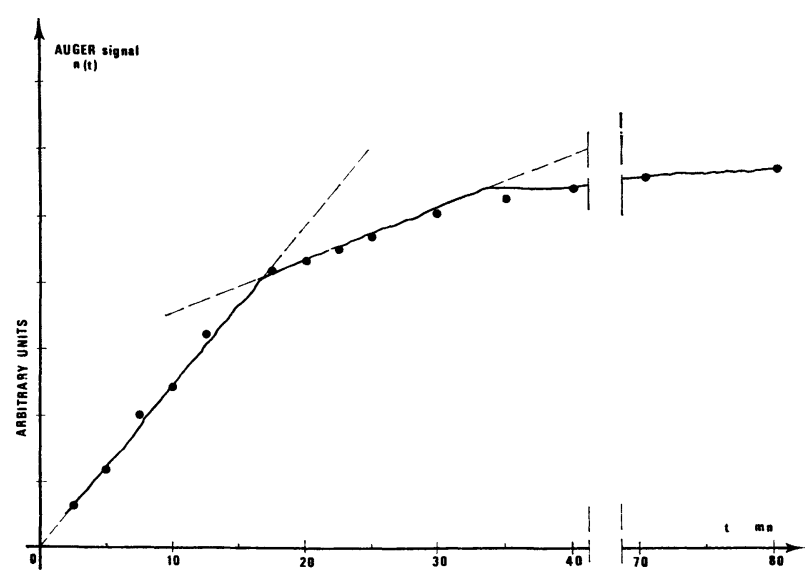

Fig. 7. - Auger amplitude of cesium as a function of deposition time for $\mathrm{Cs} / \mathrm{GaAs}$ (110). The points are obtained by the classical method while the line is obtained using the technique described in the text.

intensity is attenuated by the second layer and the total intensity of the two layers is not the double of that of a monolayer. Hence we see a change in slope of the calibration curve.

We note here that the time necessary for a second layer deposition is equal to that of the first layer. All these experimental facts prove that the adsorption of $\mathrm{Cs}$ on (110) $\mathrm{GaAs}$ procedes layer by layer and that the sticking coefficient remains constant for the first layers $\left({ }^{1}\right)$.

As the proportionality coefficient between the deposited quantity and the Auger peak height is different for successive layers, one must make a normalization to analyse correctly several experiments with different initial deposited quantities.

3.4 TherMal DESORPTION AND RESUlts. - After having deposited an initial Cs quantity, the sample is heated rapidly with a linear temperature rise $T(t)$ of about $2 \% / \mathrm{s}$. The temperature $T(t)$ is recorded by a chromel alumel thermocouple attached to the crystal surface. $\frac{\mathrm{d} n(t)}{\mathrm{d} t}$ and $n(t)$ are detected simultaneously with our apparatus monitoring the $47 \mathrm{eV}$ Cs peak.

Figure 8 is a drawing of the untreated signals of an experiment with an initial deposit of $n \simeq 8 \times 10^{14}$ atoms $/ \mathrm{cm}^{2}$. These recordings, without normalization have an acceptable signal to noise ratio.

Figure 9 shows some selected thermal desorption curves $\frac{\mathrm{d} n(t)}{\mathrm{d} t}$ for different initial coverages, versus temperature $T$. These curves have been normalized, according to the precedent calibration. In this figure, we see that for initial coverages $n<\sim 3 \times 10^{14}$ atoms $/ \mathrm{cm}^{2}$, the desorption spectrum is characterized

(1) Other experiments, not shown here, with higher Cs incident flux, allowed us to observe four well marked layers of Cs during its growth on (110) GaAs maintained at $50^{\circ} \mathrm{C}$. 


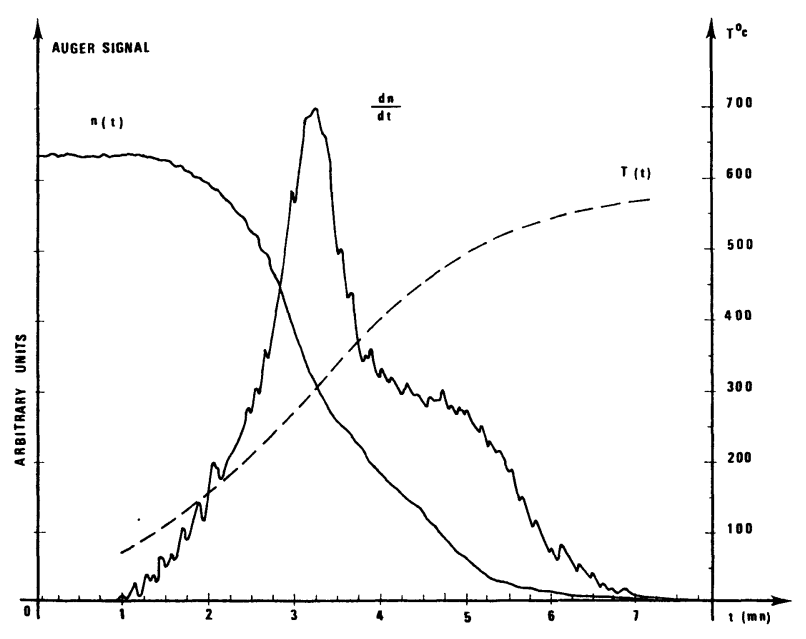

FIG. 8. - Recordings of thermal desorption experiment with our technique before normalization.

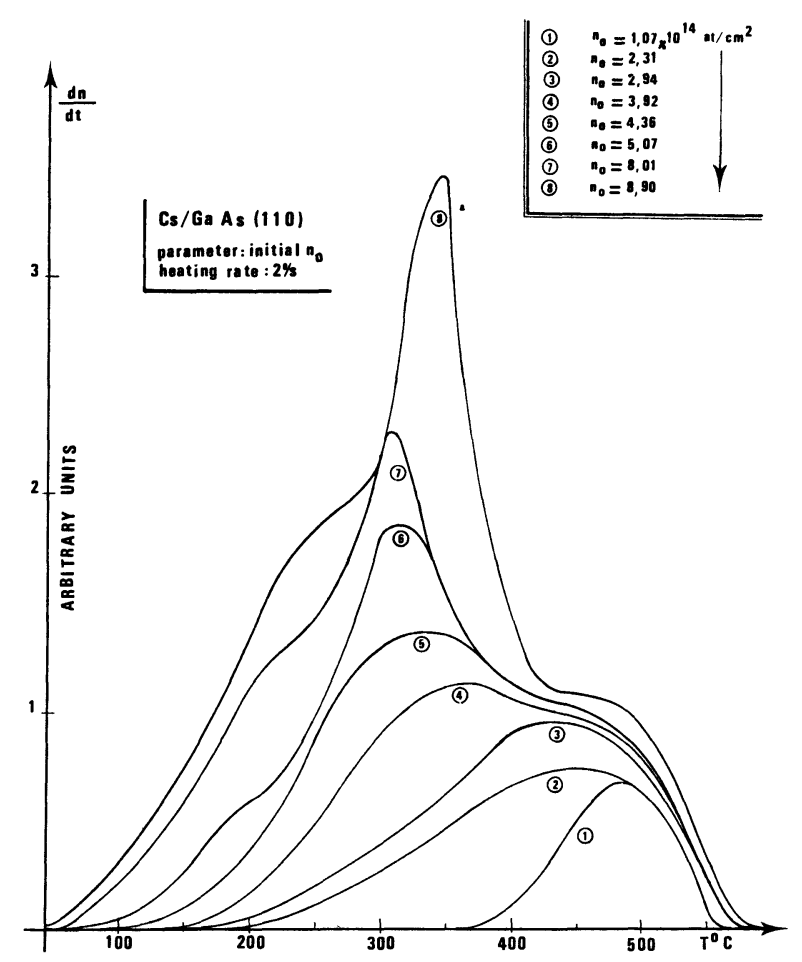

FIG. 9. - Desorption spectra of cesium adsorbed on (110) GaAs using modified Auger spectrometer.

by a single high temperature peak at about $500^{\circ} \mathrm{C}$. For $n>\sim 3 \times 10^{14}$ atoms $/ \mathrm{cm}^{2}$, a second peak appears with its maximum at about $300^{\circ} \mathrm{C}$. This peak has a shoulder near $200^{\circ} \mathrm{C}$ which grows with increasing initial deposits.

These results show that Cs adsorption on (110) GaAs exhibits several distinct phases which can be summarized in the following way :

For small coverages, Cs is strongly bound to the GaAs surface. There is a partial electronic charge transfert from $\mathrm{Cs}$ to $\mathrm{GaAs}$. Electrostatic dipoles $\mathrm{Cs} / \mathrm{GaAs}$ are created at the surface and these dipoles lower the work function $[10,12,14,15]$. The high temperature of the desorption peak $\left(\sim 500^{\circ} \mathrm{C}\right)$ is an indication of the strong nature of the bond. With increasing coverage, the repulsive energy between the partially ionized Cs adatoms increases. Thus the binding energy decreases, which manifests itself in the low temperature desorption peak group $\left(\sim 200^{\circ}-300^{\circ} \mathrm{C}\right)$. This situation is quite similar for alkali metals on metals [21].

Our desorption curves, from cesiated (110) GaAs, resemble, qualitatively, the Golstein's curves obtained, for (100) face [18]. However, a slight difference exists for the low temperature peak group.

This discrepancy may be explained by the two different substrate surfaces. In our case the (110) face is stable $(1 \times 1$ structure in L. E. E. D. diagram). It has the same number of $\mathrm{Ga}$ and As atoms. The clean (110) surface potential, as seen by adsorbed Cs atoms is probably different from that of the clean (100) face which is unstable and shows a reconstruction in the L. E. E. D. diagram $(2 \times 8$ superstructure $)$. Moreover, Cs adsorbs on (100) GaAs as an amorphous layer [6] while it grows epitaxially on (110) GaAs [12, 13].

Due to the lack of information on the pre-exponential factor $v(n)$ in eq. (1).

$$
-\frac{\mathrm{d} n(t)}{\mathrm{d} t}=v(n) n(t) \exp -\frac{E_{\mathrm{d}}(n)}{k T(t)}
$$

we give here an estimation only of the desorption energy for $\mathrm{Cs} /(110) \mathrm{GaAs}$ versus coverage (Table I).

\section{TABLE I}

\begin{tabular}{|c|c|}
\hline $\begin{array}{l}n \\
10^{14} \text { atoms } / \mathrm{cm}^{2}\end{array}$ & $\underset{\mathrm{eV}}{\text { Energy }} E_{\mathrm{d}}$ \\
\hline$\overline{0.44}$ & $\overline{2.17}$ \\
\hline $\begin{array}{l}0.44 \\
0.80\end{array}$ & $\begin{array}{l}2.17 \\
2.12\end{array}$ \\
\hline 1.24 & 2.10 \\
\hline 1.59 & 1.98 \\
\hline 1.95 & 1.90 \\
\hline 2.39 & 1.76 \\
\hline 2.83 & 1.70 \\
\hline 3.36 & 1.62 \\
\hline 3.89 & 1.55 \\
\hline 4.60 & 1.46 \\
\hline
\end{tabular}

A first order desorption equation was used and this assumption seems quite plausible according to Golstein's work [18].

Energies $E_{\mathrm{d}}(n)$ were calculated from eq. (1) assuming $v=10^{13} \mathrm{~s}^{-1}$ and using the experimental normalized values $n(t)$ and $\frac{\mathrm{d} n(t)}{\mathrm{d} t}$, and the plots $T(t)$. The results listed in table $I$ are average values from several desorption curves of figure 9. The fluctuations $\Delta E_{\mathrm{a}}$ 
are about $0.08 \mathrm{eV}$. Experimental $v$ values given in the literature for alkali metals are in the range

$$
10^{11} \mathrm{~s}^{-1}<v<10^{15} \mathrm{~s}^{-1} \text {. }
$$

If we take these extreme values for calculating desorption energies, we find that $E_{\mathrm{d}}(n)$ are shifted by about $\pm 0.2 \mathrm{eV}$.

A more detailed study of both $E_{\mathrm{d}}(n)$ and $v(n)$ is envisaged using other experiments, complementary to thermal desorption (measurement of isosteric adsorption heats, measurement of adsorption and desorption kinetics with modulated flux).

4. Discussion. - It has been shown in the previous chapters that a modified Auger spectrometer is a suitable means for following the kinetics of surface reactions. This method is equivalent to the use of a high performance mass spectrometer in desorption kinetics. Its sensitivity is that of an Auger spectrometer $\left(\sim 10^{12}\right.$ atoms $\left./ \mathrm{cm}^{2} / \mathrm{s}\right)$. With an A. E. S. probing area of $1 \mathrm{~mm}^{2}$, one can detect a variation of about $10^{10}$ atoms/s. This technique has several advantages :

- Contrary to mass spectrometry, the adatom population is directly measured on the surface. Thus shields, as used with the former technique in order to eliminate stray desorption from all possible parts of the sample holder are not needed.

A. E. S., in this case, gives direct results from the surface itself as in a field electron emission technique [22].

- It is possible to follow, directly on the surface, the rate of chemical compound formation, superficial segregation...

- Another advantage is the low cost set up which increases the already numerous applications of an Auger spectrometer. Such an instrument is, for many surface studies, indispensable.
These potentials are to be balanced against some shortcomings :

- The signal to noise ratio is less favourable than that of a high performance mass spectrometer. This is mainly due to the Auger signal itself which represents variations of $\sim 10^{-9} \mathrm{~A}$ on a background of $\sim 10^{-6} \mathrm{~A}$.

- Cares must be taken to verify that the intrinsic Auger transition yield dœs not vary with the temperature. Within the experimental error, we have not observed such changes during the course of our experiments.

- For high precision measurements, it is necessary to choose Auger transitions whose peak energies do not depend on the atom concentration.

- Using this technique, one can only study surface reactions involving chemical elements different from those of the substrate.

5. Conclusion. - In the first part of this paper, we have proposed a new technique for monitoring surface reaction kinetics in ultra high vacuum, using Auger Spectroscopy.

In the second part of the paper, we have applied this method to an investigation of the adsorption (and desorption) of cesium on (from) (110) gallium arsenide. The following results are obtained : Cs adsorption procedes in a layer by layer fashion. For small coverages, several binding states are found and this result is consistent with those obtained elsewhere by photoemission and work function measurements.

Acknowledgements. - The authors are grateful to Pr. M. Bienfait for helpful and stimulating discussions, and to Dr. G. Poiblaud (R. T. C., Caen) for providing GaAs crystals.

\section{References}

[1] Chang, C. C., Surf. Sci. 25 (1971) 53.

[2] Review paper concerning the thermal desorption by Petermann, L. A., progress in Surf. Sci. 3 (1972) (Pergamon Press).

[3] Weber, R. E. and Johnson, A. L., J. Appl. Phys. 40 (1969) 314.

[4] Guillot, C., 3rd cycle Thesis, Université Paris Sud, Orsay (1972).

[5] Pichon, J. L., 3rd cycle Thesis, Université Aix-Marseille (1973).

[6] Goldstein, B., Surf. Sci. 47 (1975) 155

[7] Stocker, B. J., Surf. Sci. 47 (1975) 501.

[8] Gillet, E. et al., to be published on Surf. Sci.

[9] Sickafus, E. N. and Colvin, A. D., Rev. Sci. Instrum. 41 (1970) 1349.

[10] Scheer, J. J. and van LaAR, J., Solid State Commun. 5 (1967) 740.
[11] VAn LaAR, J., Acta Electron. 16 (1973) 215, and BeLL, R. L. Negative electron affinity devices (Clarendon-Oxford) 1973.

[12] Yu Mityagin, A. et al., Sov. Phys. Solid State 14 (1973) 1623.

[13] Van Bommel, A. J. and Crombeen, J. E., Surf. Sci. 45 (1974) 308.

[14] Derrien, J. et al., Surf. Sci. 47 (1975) 162.

[15] MadeY, T. E. and YATES, J. T. Jr, J. Vacuum Sci. Techn. 8 (1971) 39.

[16] Fisher, T. E., Phys. Rev. 147 (1966) 603.

[17] Sмith, D. L. and Huchital, D. A., J. Appl. Phys. 43 (1972) 2624.

[18] Goldstein, B., Appl. Phys. Lett. 26 (1975) 111.

[19] Weber, R. E. and CoRDEs, L., Rev. Sci. Instrum. 37 (1966) 112.

[20] KleIN, W., Rev. Sci. Instrum. 42 (1971) 1082.

[21] Langmuir, J., J. Amer. Chem. Soc. 54 (1932) 2798.

[22] Schmidt, L. and Gomer, R., J. Chem. Phys. 42 (1965) 3573. 